\title{
BMJ Open Knowledge acquisition and retention after a high flow training programme in Peru: a quasi-experimental single group pre-post design
}

\author{
Laura E Ellington (D) , ${ }^{1}$ Rosario Becerra, ${ }^{2}$ Gabriela Mallma, ${ }^{2}$ \\ José Tantaleán da Fieno, ${ }^{2,3}$ Deepthi Nair, ${ }^{4}$ Frankline Onchiri, ${ }^{4}$ Katie R Nielsen (1D) ${ }^{5,6}$
}

To cite: Ellington LE, Becerra R, Mallma G, et al. Knowledge acquisition and retention after a high flow training programme in Peru: a quasiexperimental single group pre-post design. BMJ Open 2020;10:e035125. doi:10.1136/ bmjopen-2019-035125

- Prepublication history and additional material for this paper are available online. To view these files, please visit the journal online (http://dx.doi. org/10.1136/bmjopen-2019035125).

Received 21 October 2019 Revised 07 May 2020 Accepted 14 May 2020
Check for updates

(C) Author(s) (or their employer(s)) 2020. Re-use permitted under CC BY-NC. No commercial re-use. See rights and permissions. Published by BMJ.

For numbered affiliations see end of article.

Correspondence to

Dr Katie R Nielsen;

ktruth@uw.edu

\section{ABSTRACT}

Objective Respiratory infections remain the leading infectious cause of death in children under 5 and disproportionately affect children in resource-limited settings. Implementing non-invasive respiratory support can reduce respiratory-related mortality. However, maintaining competency after deployment can be difficult. Our objective was to evaluate the effectiveness of a comprehensive multidisciplinary high-flow training programme in a Peruvian paediatric intensive care unit (PICU).

Design Quasi-experimental single group pre-post intervention study design.

Setting Quaternary care PICU in a resource-constrained setting in Lima, Peru.

Participants Attending physicians, fellows, paediatric residents, registered nurses, respiratory therapists and medical technicians working in the PICU were invited to participate.

Interventions Concurrent with initial high-flow deployment, we implemented a training programme consisting of lectures, case-based discussion and demonstrations with baseline, 3-month and 12-month training sessions. Pre-training and post-training assessment surveys were distributed surrounding all training sessions.

Primary and secondary outcome measures The primary outcome was achieving minimum competency (median score of $80 \%$ ) on the high flow training assessment tool. Secondary outcomes included knowledge acquisition (differences in pre-baseline and post-baseline training assessments), short-term retention (differences in post-baseline and pre-3-month refresher training assessments) and long-term retention (differences in post3-month refresher and pre-12-month refresher training assessments).

Results Eighty participants (50\% nurses, $15 \%$ ICU physicians and $34 \%$ other providers) completed the baseline assessment. Participants showed improvement in overall score and all subtopics except the clinical application of knowledge after baseline training $(p<0.001)$. Participants failed to retain minimum competency at 3-month and 12-month follow-up assessments (70\% (IQR: 57-74) and 70\% (IQR: 65-74), respectively). After repeat training sessions, overall knowledge continued to improve, exceeding baseline performance (78\% (IQR: 70-87), 83\%
Strengths and limitations of this study

- Our study is the first to evaluate the impact of repeat training sessions on short-term and long-term retention in a resource-constrained setting.

- We targeted a multidisciplinary approach to high flow training as part of a larger deployment project.

- We did not specifically exclude participants who had not received prior training as part of our real-world approach.

- Because training assessments were completed without unique identifiers, we were unable to track individual progress over time but rather evaluated overall knowledge acquisition and retention on a group level.

- High provider attrition in refresher training sessions limited statistical power and introduced potential selection bias.

(IQR: 74-87) and 87\% (IQR: 83-91) at baseline, 3 and 12 months, respectively).

Conclusion This study suggests the need for repeat training sessions to achieve and maintain competency after the implementation of new technology.

\section{INTRODUCTION}

Respiratory infections remain the leading infectious cause of death in children under 5 , and children in resource-limited settings (RLS) are disproportionately affected. ${ }^{1}$ Increased availability of non-invasive respiratory support, such as continuous positive airway pressure (CPAP) and nasal high flow (high flow), in RLS has likely contributed to the $20 \%$ decrease in mortality from lower respiratory tract infections in this age group. ${ }^{1}$ Outcomes following the deployment of respiratory technology have shown variable success, ${ }^{2}$ and concerns related to safety and sustainability in these settings have arisen. ${ }^{34}$

One important factor for the successful implementation and sustainability of 
respiratory technology in RLS is the presence of a highquality training programme. ${ }^{5}$ Current data suggest that knowledge and skills retention after initial training programmes decline over time, ${ }^{6-11}$ highlighting the need for ongoing refresher training in order to sustain competency. As part of the initial deployment of high flow in the paediatric intensive care unit (PICU) at Instituto Nacional de Salud del Niño (INSN) in 2016, we developed a comprehensive multidisciplinary high flow training programme that included initial and refresher training sessions for all PICU staff. The objective of this study was to evaluate the effectiveness of our high flow training programme by determining participant knowledge acquisition and retention over time. We hypothesised that training programme participants would achieve competency and demonstrate short-term and longterm knowledge retention through scheduled refresher training and clinical experience.

\section{METHODS}

\section{Design}

We performed a quasi-experimental single group prepost interventional study, introducing a high flow training programme with scheduled refresher training sessions at three discrete time points: baseline, 3 months and 12 months.

\section{Setting and participants}

INSN is the largest quaternary care children's hospital in Peru. Its basic demographics and PICU characteristics have been previously described. ${ }^{5}{ }^{12}$ Prior to this study, high flow was not available at INSN. Available respiratory support included invasive mechanical ventilation and non-invasive positive pressure ventilation via CPAP. CPAP was infrequently used due to patient discomfort, difficulties maintaining an adequate seal and skin breakdown. The research team designed a pilot implementation project of high flow within the PICU based on recommendations from INSN PICU providers. Prior to high flow deployment, PICU staff received high flow training according to our comprehensive multidisciplinary training programme.

Subjects were eligible for participation in this study if they were working clinically in the PICU at the time of a high flow training session. We did not exclude participants if they had not attended previous training sessions. Participants included PICU attending physicians, PICU fellows, paediatric residents on their PICU rotation, registered nurses working exclusively in the PICU, respiratory therapists and medical technicians.

\section{Intervention}

A multidisciplinary high flow training programme was implemented that included sessions prior to initial high flow deployment and at 3-month and 12-month follow-up intervals. Baseline training consisted of didactic lecture and hands-on demonstration of the high flow system. The didactic lecture was adapted from a high flow training programme used at authors LEE and KRN's institution, using the evidence-based literature. Baseline training was performed two times per day for four consecutive days during clinical hours to maximise PICU staff participation. Each day, PICU nurses arranged coverage so that half could attend the morning session and the other half could attend the afternoon session. Based on participant feedback from initial sessions, follow-up refresher training incorporated case-based discussion following the didactic lecture. Refresher trainings at 3 and 12 months were performed daily for four consecutive days during clinical hours. No nursing coverage was arranged, so refresher training took place within the clinical space so that more nurses could attend. All training sessions lasted approximately $90 \mathrm{~min}$. The didactic lecture, high flow system set-up video and protocol-specific visual aides were available on the hospital computer for just-in-time training throughout the study period.

Authors LEE and KRN developed assessment surveys to reflect learning objectives from the didactic lecture (online supplementary appendix 1). Surveys were piloted with a convenience sample of seven physicians in paediatric critical care, paediatric emergency medicine and general paediatrics at the two authors' institutions, all of whom used high flow in their clinical practice. The assessment tool was translated into Spanish and reviewed by authors RB, GM and JT for grammar, clarity and cultural appropriateness. No formal reliability or validity assessment was performed prior to deployment. Questions evaluated participant understanding of the following subtopics: high flow indications and contraindications, protocol-specific details, clinical signs of high flow failure, potential adverse events and clinical application of knowledge. For questions with multiple correct answers, participants received one point for identifying each correct answer and one point for identifying each incorrect answer. There were 23 total points possible: 7 points for indications/contraindications, 2 points for protocol-specific details, 7 points for signs of high-flow failure, 5 points for potential adverse events and 2 points for clinical application of knowledge (online supplementary appendix 2).

\section{Data collection}

Pre-training and post-training assessment surveys were distributed immediately prior to and following each training session (online supplementary appendices 1 and 2). Protocol-specific visual aids with the high flow protocol were available to participants during post-training assessment surveys and have been published elsewhere. ${ }^{12}$

\section{Outcomes}

The primary outcome of interest was achieving minimum competency on the high flow training assessment tool. We defined minimum competency as achieving a median score of $80 \%$. Secondary outcomes included knowledge acquisition and short-term and long-term knowledge 
retention. Knowledge acquisition was defined as improvement in median score from baseline pre-assessment to post-assessment. Short-term knowledge retention was defined as no difference in median score between postbaseline and pre-3-month refresher assessment. Longterm knowledge retention was defined as no difference between post-3-month refresher and pre-12-month refresher assessment.

\section{Statistical analysis}

The basic demographics of survey respondents were summarised using counts (and proportions). Participant performance was summarised using median (IQR) for overall score and for subtopics outlined above. We evaluated knowledge acquisition and short-term and long-term retention by comparing pre-training and posttraining scores using the Wilcoxon rank sum test (a nonparametric test alternative to a t-test), as the data were not all normally distributed according to the Shapiro-Wilk normality test. Analyses were conducted using STATA statistical software (V.14.2).

Given that paediatric residents rotate in the PICU for 1-2 months duration and would not be available for assessment of knowledge retention, we performed post hoc sensitivity analyses to determine whether their exclusion from knowledge retention analyses influenced results. Due to differences in short-term and long-term retention analyses, we report retention results that exclude paediatric residents.
Patient and public involvement statement

No patients were involved in this study.

\section{RESULTS}

Eighty participants completed the baseline pre-training assessment: 40 registered nurses (50\%), 15 medical technicians (19\%), 12 PICU-trained physicians (15\%), 10 paediatric residents $(13 \%)$ and 2 respiratory therapists $(3 \%)$. Sixty-six percent of participants had $>10$ years' experience as a healthcare provider and $44 \%$ had $>10$ years' ICU experience. There was $>50 \%$ drop off in refresher training participation, with only 27 individuals completing the 3-month pre-refresher training assessment and 36 completing the 12-month pre-refresher training assessment (table 1).

Participants showed statistically significant improvement in overall score and all subtopics except the clinical application of knowledge after baseline training $(p<0.001)$ (figure 1). After baseline training, participants failed to achieve minimum competency in overall score (78\% (70-87)), but achieved minimum competency in all subtopic areas except the clinical application of knowledge (table 2). Participant overall performance exceeded baseline performance after 3-month and 12-month refresher training sessions, (78\% (70-87), $83 \%$ (74-87) and $87 \%(83-91)$ at baseline, 3 and 12 months, respectively), demonstrating incremental improvement and achieving minimum competency after 3-month and 12-month refresher training sessions. Clinical application

Table 1 Participant characteristics for each training session

\begin{tabular}{|c|c|c|c|c|c|c|}
\hline & \multicolumn{2}{|c|}{ Baseline training } & \multicolumn{2}{|c|}{$\begin{array}{l}\text { 3-month refresher } \\
\text { training }\end{array}$} & \multicolumn{2}{|c|}{$\begin{array}{l}12-\text { month refresher } \\
\text { training }\end{array}$} \\
\hline & Pre & Post & Pre & Post & Pre & Post \\
\hline $\mathrm{n}$ & 80 & 76 & 27 & 32 & 36 & 27 \\
\hline \multicolumn{7}{|l|}{ Provider role, $\mathrm{n}(\%)^{*}$} \\
\hline ICU-trained physician (fellow or attending) & $12(15)$ & $12(16)$ & $4(15)$ & $4(13)$ & $2(6)$ & $3(11)$ \\
\hline Paediatric resident & $10(13)$ & $8(11)$ & $8(30)$ & $11(34)$ & $3(8)$ & $2(7)$ \\
\hline Registered nurse & $40(50)$ & $40(53)$ & $14(52)$ & $14(44)$ & $27(75)$ & $19(70)$ \\
\hline Respiratory therapist & $2(3)$ & $1(1)$ & 0 & 0 & 0 & 0 \\
\hline Medical technician & $15(19)$ & $14(18)$ & $1(4)$ & $1(3)$ & $4(11)$ & $3(11)$ \\
\hline \multicolumn{7}{|l|}{ Years of ICU experience, $\mathrm{n}(\%)$} \\
\hline$>10$ years & $35(44)$ & $30(39)$ & $13(48)$ & $15(47)$ & $21(58)$ & $13(48)$ \\
\hline \multicolumn{7}{|l|}{ Years as healthcare provider, $\mathrm{n}(\%)$} \\
\hline$>10$ years & $53(66)$ & $45(59)$ & $16(59)$ & $18(56)$ & $27(75)$ & $15(56)$ \\
\hline \multicolumn{7}{|l|}{ High-flow usage, n (\%) } \\
\hline Never & $52(65)$ & $66(87)$ & $19(70)$ & $23(72)$ & 7 (19) & $9(33)$ \\
\hline $1-5$ times & $10(13)$ & $5(7)$ & $7(26)$ & $9(28)$ & $20(56)$ & $13(48)$ \\
\hline$>5$ times & $13(16)$ & $3(4)$ & $1(4)$ & 0 & $7(19)$ & $5(19)$ \\
\hline
\end{tabular}

*Missing provider role data: baseline pre $(n=1)$, baseline post $(n=1), 3$-month post $(n=2)$.

ICU, intensive care unit. 


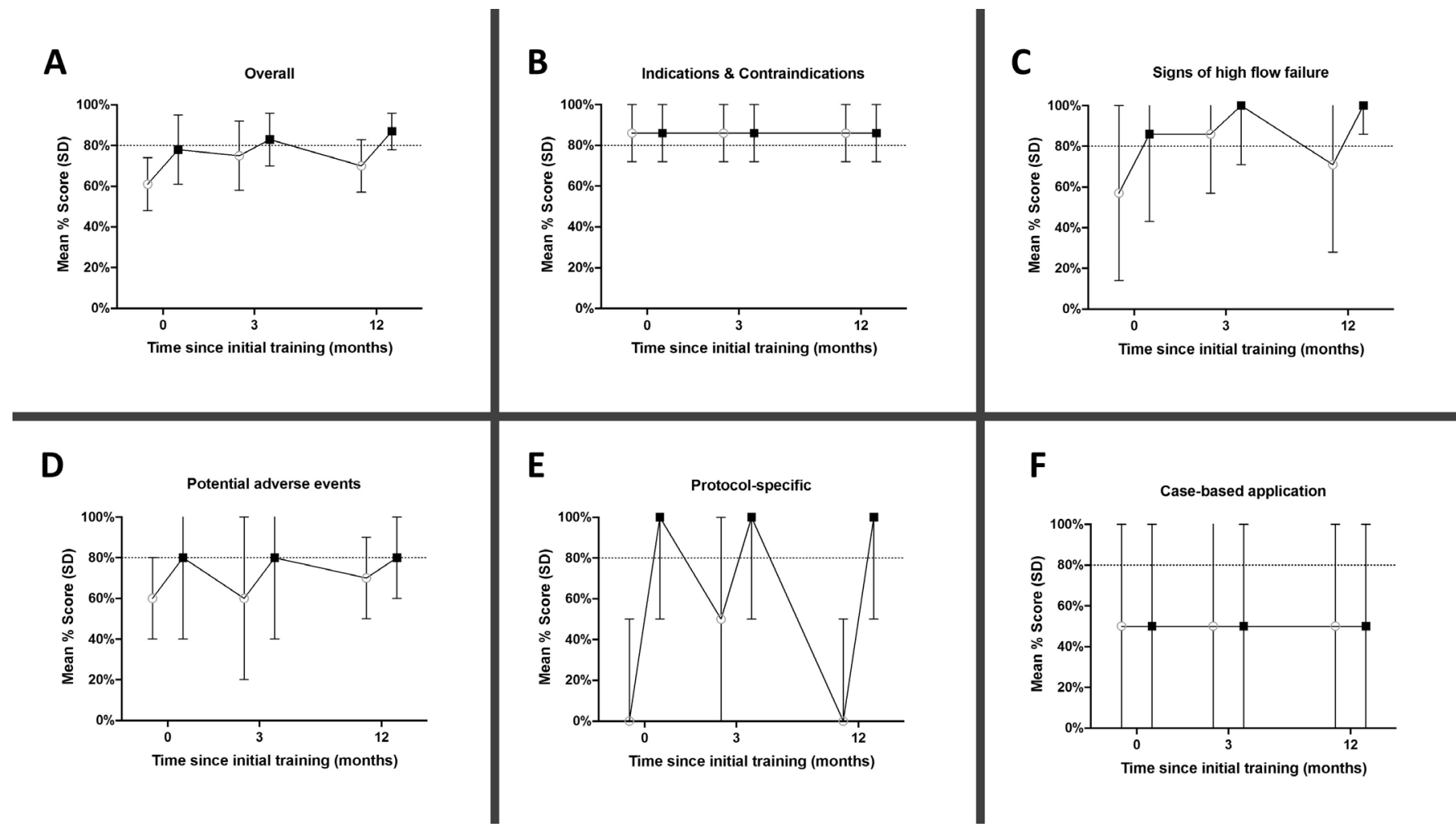

Figure 1 Provider performance on pre-assessment and post-assessments during the study period. Baseline training occurred at time 0 with refresher training sessions 3 and 12 months after baseline training. Open circles illustrate pre-training assessment, and closed squares demonstrate post-training assessment. Error bars represent SD. Minimum competency was defined by a minimum score of $80 \%$ (horizontal dashed line). All providers, including paediatric residents, are included in this figure. Differences in pre-baseline and post-baseline assessments determined knowledge acquisition. Acquisition was attained overall and for all subtopics except clinical application of knowledge $(p<0.001)$. Participants did not achieve short-term (from postbaseline to pre-3-month refresher training assessments) or long-term (from post-3-month refresher to pre-12-month refresher training assessments) retention in the overall scores $(p<0.05)$. Long-term retention was not achieved in any subtopic except high flow indications/contraindications $(\mathrm{p}=0.09)$. All comparisons were performed using the Wilcoxon rank-sum test.

of knowledge performance was low throughout the study period regardless of training. Scores from all training sessions are reported in tables 2 and 3.

Overall short-term and long-term retention suffered throughout the study period, with participants failing to retain minimum competency at 3-month and 12-month follow-up assessments (74\% (61-78) and 70\% (63-76), respectively). This poor retention correlated with infrequent high flow use during the study period (table 1). In the short- and long-terms, participants retained minimum competency only in the high flow indications/contraindications subtopic. In the long-term, participants also retained competency in adverse events (figure 1, table 3 ).

\section{DISCUSSION}

In this study, we found that participants in our high flow training programme demonstrated knowledge acquisition after baseline training and achieved minimum competency in all subtopic areas except the clinical application of knowledge after refresher training sessions. Short-term and long-term knowledge retention was suboptimal, with failure to retain minimum competency at 3 and 12 months. Despite the loss of knowledge, refresher training sessions helped participants achieve competency, with overall scores exceeding those after baseline training. Our findings suggest the importance of repeat training sessions in order to achieve and maintain competency after the implementation of new technology.

Other studies have reported challenges with knowledge retention after training programmes at intervals ranging from 3 to 12 months after initial training. ${ }^{6-11}$ All reported varying degrees of knowledge loss over time, but none performed interim refresher training but rather relied on clinical experience to reinforce concepts between assessment's time points. Two studies assessing the effect of cardiopulmonary resuscitation (CPR) refresher training reported improved participant confidence ${ }^{13}$ and time to obtain skill success. ${ }^{14}$ Our study provides evidence for an additional benefit of refresher training - to continue to build on participants' existing knowledge base, particularly in settings where clinical use is low.

Despite this success, this study had several limitations, identifying areas for improvement of our training programme. First, we observed substantial attrition in attendance at refresher training sessions, which had fewer than 50\% the number of participants as baseline training. Attrition 


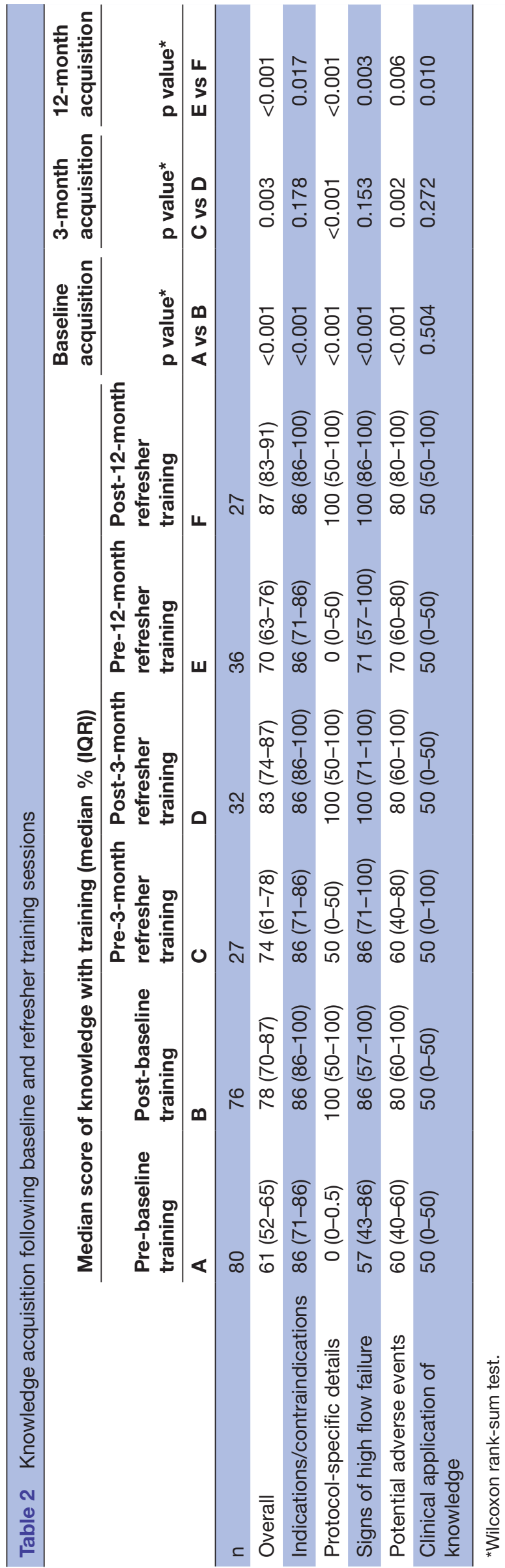

in provider participation limited statistical power in our study and may have introduced selection bias. With only 27-36 participants completing post-enrolment assessment, the study likely lacked sufficient power to detect anything but large effects in knowledge acquisition and retention. Despite this limitation, most of the pairwise comparisons for knowledge acquisition were statistically significant, suggesting the lack of power may not have been a major issue. However, insufficient power may have affected retention analyses by decreasing our ability to detect differences and therefore concluding that the lack of difference was consistent with knowledge retention.

There are several potential explanations for this drop off in participation. Although efforts were made to maximise provider participation by scheduling training sessions around the nurses' clinical schedules, due to resource constraints, we were not able to offer the same number of refresher training sessions as we did with the initial training. Furthermore, the combination of a lack of protected time for non-clinical activities and the non-mandatory nature of training likely influenced attendance. In addition, the interdisciplinary nature of our training programme may have made it less desirable for some providers, given variable learning expectations among different provider groups. Other multidisciplinary training programmes have observed differential results according to provider role, ${ }^{15}$ suggesting that providing separate training programmes tailored to the needs of different provider groups may improve attendance by increasing relevance of content and impact performance on competency assessments.

An additional limitation was the high variability of performance in different subtopic areas, which may be due to the assessment tool itself. The questions ranged from direct questions with a single correct answer to application questions in which multiple answers were correct. We attempted to account for the varying difficulty by giving marks for both correct and incorrect responses to give more weight to complex questions and reward participants for knowing when high flow would not be appropriate to use. However, we acknowledge that this scoring system may have skewed results. Participants in the pilot testing did not express concerns about or struggle with the different questions types; however, all pilot participants were physicians. It is possible that the format of the questionnaire could have been challenging for training participants to understand and may have impacted performance. Including other provider roles in the pilot would have been helpful. In addition, each subtopic had a range of potential points available, from 2 to 7 . Therefore, maintaining competency in each subtopic (at least $80 \%$ ) would be difficult if possible scores are $0 \%, 50 \%$ or $100 \%$. That said, our data suggest that some subtopics were easier to learn and retain than others.

Another limitation was the low frequency of high flow use during the study period, which could have influenced participants' ability to remember practical details of high flow use, independent of the training programme. 
Table 3 Short-term and long-term knowledge retention following baseline and refresher training sessions excluding paediatric residents

\begin{tabular}{|c|c|c|c|c|c|c|}
\hline & $\begin{array}{l}\text { Median score } \\
\text { (median \% (IQF }\end{array}$ & $\begin{array}{l}\text { ff knowledge n } \\
\text { 2)) }\end{array}$ & vith training & & $\begin{array}{l}\text { Short-term } \\
\text { retention }\end{array}$ & $\begin{array}{l}\text { Long-term } \\
\text { retention }\end{array}$ \\
\hline & $\begin{array}{l}\text { Post-baseline } \\
\text { training }\end{array}$ & $\begin{array}{l}\text { Pre-3-month } \\
\text { refresher } \\
\text { training }\end{array}$ & $\begin{array}{l}\text { Post-3-month } \\
\text { refresher } \\
\text { training }\end{array}$ & $\begin{array}{l}\text { Pre-12-month } \\
\text { refresher } \\
\text { training }\end{array}$ & p value* & $p$ value \\
\hline & B & C & D & $\mathbf{E}$ & B vs C & D vs E \\
\hline $\mathrm{n}$ & 67 & 19 & 19 & 33 & & \\
\hline Overall & $78(70-87)$ & $70(57-74)$ & $83(74-87)$ & $70(65-74)$ & 0.017 & 0.001 \\
\hline Indications/contraindications & $86(86-100)$ & $86(71-86)$ & $86(86-100)$ & $86(71-86)$ & 0.180 & 0.097 \\
\hline Protocol-specific details & $100(50-100)$ & $50(0-50)$ & $100(50-100)$ & $0(0-50)$ & $<0.001$ & $<0.001$ \\
\hline Signs of high-flow failure & $86(57-100)$ & $71(57-100)$ & $100(71-100)$ & $71(57-100)$ & 0.447 & 0.126 \\
\hline Potential adverse events & $80(60-100)$ & $60(40-80)$ & $80(60-100)$ & $80(60-80)$ & 0.001 & 0.427 \\
\hline Clinical application of knowledge & $50(0-50)$ & $50(0-100)$ & $50(50-50)$ & $50(0-50)$ & 0.088 & 0.992 \\
\hline
\end{tabular}

*Wilcoxon rank-sum test.

Previous studies suggested that high volume (or lack thereof) contribute to retained competency following a training programme, ${ }^{16} 17$ emphasising the importance of evaluating a healthcare setting's patient volume prior to introducing new technology. Prior to this training programme, we did not anticipate low high flow use. The high proportion of children with chronic comorbid conditions and/or other exclusion criteria for the pilot implementation research study decreased the volume of candidates for high flow during the study period. Given the low frequency of clinical use of high flow, just-intime training was available on the local computer, but its usage by staff was not assessed. Altogether, these findings underscore the difficulties in retaining information in the setting of the low frequency of use. This could represent an opportunity for the introduction of simulation to reinforce practical aspects of care delivery.

Finally, despite our efforts to collect unique identifiers for study participants in order to track performance over time, this portion of the questionnaire was infrequently completed by participants. This made it impossible to perform a paired analysis. Given that some individuals likely completed assessments at multiple time points, the populations were not completely independent, which is an assumption of the Wilcoxon rank-sum test. A more appropriate test would have been the Wilcoxon signed-rank test, which we would have performed if we had successfully tracked individuals at all time points throughout the training programme and paired the data.

One concerning finding was participants' poor retention in identifying potential adverse events associated with high flow use. These included life-threatening complications, such as pneumothorax, which must be promptly recognised to prevent catastrophic outcomes. These results prompted us to reconsider the goals of our training programme. Although disseminating general knowledge about high flow is an essential component of a training programme, most of these details could be provided in a variety of educational handouts or modules at the bedside. However, helping providers better recognise life-threatening complications has the potential to reduce preventable harm and adverse clinical outcomes. Emphasising these serious adverse events in the take-away points of the training module and/or including a case-based example of a child with a pneumothorax could help raise awareness of these life-threatening complications and improve retention over time.

Given the limited power of our study, particularly in subtopic areas, future directions should include efforts to improve provider participation in refresher training sessions to better understand knowledge retention in this setting. Furthermore, future studies evaluating training programmes should consider adding a qualitative aim to better understand participant attrition using individual interviews or focus groups with those who chose to participate and others who did not.

In conclusion, the poor retention of high flow knowledge after initial training underscores the need for frequent refresher training and robust monitoring and evaluation after implementation of new technology. Although applicable to all clinical settings, RLS face their own unique challenges, such as limitations in protected time for training, technological expertise and opportunities for clinical application, which add to known difficulty with knowledge retention. However, even in these settings, appropriately tailored, frequent refresher training programmes are effective ways to achieve and maintain competency following the deployment of new technology. By engaging local collaborators and continuously adapting training programmes to local needs, the sustainability of new technology, such as high flow, in RLS can be achieved.

Author affiliations

${ }^{1}$ Graduate Medical Education, University of Washington, Seattle, Washington, USA 
${ }^{2}$ Departamento de Cuidados Intensivos Pediátricos, Instituto Nacional de Salud del Niño, Lima, Peru

${ }^{3}$ Universidad Nacional Federico Villarreal, Lima, Peru

${ }^{4}$ Seattle Children's Core for Biomedical Statistics, Seattle Children's Research Institute, Seattle, Washington, USA

${ }^{5}$ Department of Pediatrics, Critical Care Medicine, University of Washington, Seattle, Washington, USA

${ }^{6}$ Department of Global Health, University of Washington, Seattle, Washington, USA

Acknowledgements The authors thank INSN for providing training space and A/V equipment for all training sessions.

Contributors LEE and KRN had primary responsibility for study design and protocol development, training and data collection, data analysis and interpretation, manuscript preparation and review. KRN additionally served as mentor to LEE and oversaw the entire project. RB, GM and JT participated in data collection, data interpretation and manuscript review. DN and FO participated in data analysis and manuscript review.

Funding This work was supported by Seattle Children's Center for Clinical and Translational Research Clinical Research Scholars Program and Seattle Children's Center for Clinical and Translational Research Faculty Research Support Fund.

Competing interests Author KRN received travel funds from Fisher \& Paykel to attend an international high flow conference.

Patient consent for publication Not required.

Ethics approval This investigation was approved by Seattle Children's Hospital Institutional Review Board and INSN Comité de Ética.

Provenance and peer review Not commissioned; externally peer reviewed.

Data availability statement The data that support the findings of this study are available from the corresponding author, $\mathrm{KRN}$, upon reasonable request.

Open access This is an open access article distributed in accordance with the Creative Commons Attribution Non Commercial (CC BY-NC 4.0) license, which permits others to distribute, remix, adapt, build upon this work non-commercially, and license their derivative works on different terms, provided the original work is properly cited, appropriate credit is given, any changes made indicated, and the use is non-commercial. See: http://creativecommons.org/licenses/by-nc/4.0/.

\section{ORCID iDs}

Laura E Ellington http://orcid.org/0000-0001-7904-4249

Katie R Nielsen http://orcid.org/0000-0001-6127-7167

\section{REFERENCES}

1 GBD 2016 Causes of Death Collaborators. Global, regional, and national age-sex specific mortality for 264 causes of death, 19802016: a systematic analysis for the global burden of disease study 2016. Lancet 2017;390:1151-210.
2 McCollum ED, Smith AG, Eckerle M, et al. CPAP treatment for children with pneumonia in low-resource settings. Lancet Respir Med 2017:5:924-5.

3 Chisti MJ, Salam MA, Smith JH, et al. Bubble continuous positive airway pressure for children with severe pneumonia and hypoxaemia in Bangladesh: an open, randomised controlled trial. Lancet 2015;386:1057-65.

4 Wilson PT, Brooks JC, Otupiri E, et al. Aftermath of a clinical trial: evaluating the sustainability of a medical device intervention in Ghana. J Trop Pediatr 2014:60:33-9.

5 Ellington LE, Jacob-Files E, Becerra R, et al. Key considerations prior to nasal high flow deployment in a Peruvian PICU from providers perspectives. Acta Paediatr 2019;108:882-8.

6 Charalampopoulos D, Karlis G, Barouxis D, et al. Theoretical knowledge and skill retention 4 months after a European paediatric life support course. Eur J Emerg Med 2016;23:56-60.

7 Ameh CA, White S, Dickinson F, et al. Retention of knowledge and skills after emergency obstetric care training: a multi-country longitudinal study. PLoS One 2018;13:e0203606.

8 Meaney PA, Sutton RM, Tsima B, et al. Training hospital providers in basic CPR skills in Botswana: acquisition, retention and impact of novel training techniques. Resuscitation 2012;83:1484-90.

9 Rajeswaran L, Cox M, Moeng S, Tsima BM, et al. Assessment of nurses' cardiopulmonary resuscitation knowledge and skills within three district hospitals in Botswana. Afr J Prim Health Care Fam Med 2018;10:e1-6.

10 Meaney PA, Joyce CL, Setlhare S, et al. Knowledge acquisition and retention following saving children's lives course for healthcare providers in Botswana: a longitudinal cohort study. BMJ Open 2019;9:e029575.

11 Wu S, Li R, Su W, et al. Is knowledge retained by healthcare providers after training? A pragmatic evaluation of drug-resistant tuberculosis management in China. BMJ Open 2019;9:e024196.

12 Nielsen KR, Becerra R, Mallma G, et al. Successful deployment of high flow nasal cannula in a Peruvian pediatric intensive care unit using implementation Science-Lessons learned. Front Pediatr 2018;6:85.

13 Kemery S, Kelly K, Wilson C, et al. Brief bedside refresher training to practice cardiopulmonary resuscitation skills in the ambulatory surgery center setting. J Contin Educ Nurs 2015;46:370-6.

14 Niles D, Sutton RM, Donoghue A, et al. "Rolling Refreshers": A novel approach to maintain CPR psychomotor skill competence. Resuscitation 2009:80:909-12.

15 Peter NA, Pandit H, Le G, et al. Delivering a sustainable trauma management training programme tailored for low-resource settings in East, central and southern African countries using a cascading course model. Injury 2016;47:1128-34

16 Osei-Ampofo M, Tafoya MJ, Tafoya CA, et al. Skill and knowledge retention after training in cardiopulmonary ultrasound in Ghana: an impact assessment of bedside ultrasound training in a resourcelimited setting. Emerg Med J 2018;35:emermed-2018-207716.

17 Tang JH, Kaliti C, Bengtson A, et al. Improvement and retention of emergency obstetrics and neonatal care knowledge and skills in a hospital mentorship program in Lilongwe, Malawi. Int J Gynaecol Obstet 2016;132:240-3. 